\title{
New Direct Reaction: Two-Proton Knockout from Neutron-Rich Nuclei
}

\author{
D. Bazin, ${ }^{1}$ B. A. Brown, ${ }^{1,2}$ C. M. Campbell, ${ }^{1,2}$ J. A. Church,,${ }^{1,2}$ D. C. Dinca, ${ }^{1,2}$ J. Enders, ${ }^{1, *}$ A. Gade, ${ }^{1,2}$ T. Glasmacher, ${ }^{1,2}$ \\ P. G. Hansen, ${ }^{1,2, \dagger}$ W. F. Mueller, ${ }^{1}$ H. Olliver, ${ }^{1,2}$ B. C. Perry, ${ }^{1,2}$ B. M. Sherrill,,${ }^{1,2}$ J. R. Terry, ${ }^{1,2}$ and J. A. Tostevin ${ }^{3}$ \\ ${ }^{1}$ National Superconducting Cyclotron Laboratory, Michigan State University, East Lansing, Michigan 48824, USA \\ ${ }^{2}$ Department of Physics and Astronomy, Michigan State University, East Lansing, Michigan 48824, USA \\ ${ }^{3}$ Department of Physics, School of Electronics and Physical Sciences, University of Surrey, \\ Guildford, Surrey GU2 7XH, United Kingdom
}

(Received 9 January 2003; published 30 June 2003)

\begin{abstract}
The reaction ${ }^{9} \mathrm{Be}\left({ }^{28} \mathrm{Mg},{ }^{26} \mathrm{Ne}+\gamma\right) X$ has been studied at $82 \mathrm{MeV} /$ nucleon together with two similar cases, ${ }^{30} \mathrm{Mg}$ and ${ }^{34} \mathrm{Si}$. Strong evidence that the reactions are direct is offered by the parallel-momentum distributions of the reaction residues and by the inclusive cross sections. The pattern of the partial cross sections for ${ }^{28} \mathrm{Mg}$ suggests the presence of correlations. A preliminary theoretical discussion based on eikonal reaction theory and the many-body shell model is presented. The reaction holds great promise for the study of neutron-rich nuclei.
\end{abstract}

DOI: 10.1103/PhysRevLett.91.012501

A direct reaction proceeds from an initial to a final state of a system by the selective excitation of a small number of nucleonic degrees of freedom [1,2]. Its cross section is then specified by the wave functions describing these few particles. This has made direct reactions a powerful and widely applied tool for investigating oneand two-nucleon wave functions in nuclei. The present work extends the use of direct reactions by showing that two-proton removal from a neutron-rich system at high energy proceeds as a direct reaction. This is what would be expected intuitively from the asymmetry in proton and neutron separation energies which suppresses alternative reaction paths to the $-2 p$ final state. To see this, consider the nucleus ${ }^{28} \mathrm{Mg}$ with a two-proton separation energy of $30.0 \mathrm{MeV}$, where an alternative to the direct path would be the formation of ${ }^{27} \mathrm{Na}$ in a one-proton knockout. Here, however, the channel for proton evaporation is closed up to the proton separation energy of $13.3 \mathrm{MeV}$, thus excluding the major part of the strength in this direct reaction. Even above the proton threshold, neutron evaporation (with a separation energy of $6.8 \mathrm{MeV}$ ) will be much more likely.

In the following, measurements of total and differential cross sections for formation of the reaction residues are presented. The detection of coincident gamma rays provides partial cross sections, just as in our previous work on one-nucleon knockout reactions [3-7]. This is in contrast to the work of Yoneda et al. [8], who used the reaction as a tool to populate excited levels in in-beam gamma spectroscopy. Two-proton removal is clearly a favorable reaction for producing neutron-rich nuclei; the first application in a radioactive-beam experiment was the use of the $(p, 3 p)$ reaction in 1970 [9]. The two-proton knockout $\left({ }^{11} \mathrm{Be},{ }^{9} \mathrm{He}\right)$ has recently been used by Chen $e t$ $a l$. [10]. They recognized that the reaction would be direct and would offer a favorable spectroscopic factor in a search for the lowest continuum $s$ state in ${ }^{8} \mathrm{He}+n$, two units in $Z$ beyond the neutron drip line.
PACS numbers: 21.10.Jx, 24.10.-i, 25.60.Gc, 27.30.+t

The three nuclei studied, belonging to a region of current interest $[6,11-13]$, were produced in fragmentation of a $140 \mathrm{MeV} /$ nucleon ${ }^{40} \mathrm{Ar}$ beam from the CoupledCyclotron Facility at the NSCL. The secondary beams were selected in the A1900 fragment separator [14] with the momentum acceptance reduced to $0.5 \%$ in order to permit operation of the high-resolution S800 spectrograph [15] in dispersion-matched mode. The reaction energies vary considerably because of the large energy loss in the secondary beryllium target of $375(4) \mathrm{mg} / \mathrm{cm}^{2}$ (approximately $13 \mathrm{MeV} /$ nucleon for ${ }^{28} \mathrm{Mg}$ ). The average energies were $82.3,88.5$, and $67.1 \mathrm{MeV} /$ nucleon, respectively. The reaction residues were momentum analyzed in the spectrograph and unambiguously identified by the energy loss and time of flight between the beammonitoring scintillators located in the focal plane of the A1900 and scintillators in the focal plane of the S800 spectrograph.

The results from the measurements of the residues are shown in Fig. 1. The incident beam intensities and total measuring times were for ${ }^{28} \mathrm{Mg}, 2 \times 10^{4} \mathrm{~s}^{-1}$ and $18 \mathrm{~h}$, for ${ }^{30} \mathrm{Mg}, 2 \times 10^{3} \mathrm{~s}^{-1}$ and $18 \mathrm{~h}$, and for ${ }^{34} \mathrm{Si}, 8 \times 10^{3} \mathrm{~s}^{-1}$ and $32 \mathrm{~h}$. Only a part of the parallel-momentum distribution could be detected in a single setting of the spectrograph, and the complete distribution shown for ${ }^{28} \mathrm{Mg}$ is a composite from three field settings. The inclusive cross sections given in the figures have been corrected for angular and momentum acceptance [15].

The secondary target was surrounded by the SeGA (segmented germanium array) consisting of fourteen 32-fold segmented germanium detectors [16] arranged at a distance of $20 \mathrm{~cm}$ from the target in two rings with angles of $90^{\circ}$ and $37^{\circ}$ relative to the beam axis. The segmentation allowed for an event-by-event Doppler reconstruction of the energies of the $\gamma$ rays in coincidence with the knockout residues. Pseudodata from GEANT 3 simulations [17] successfully modeled the line shapes obtained after retroconversion to the center-of-mass 


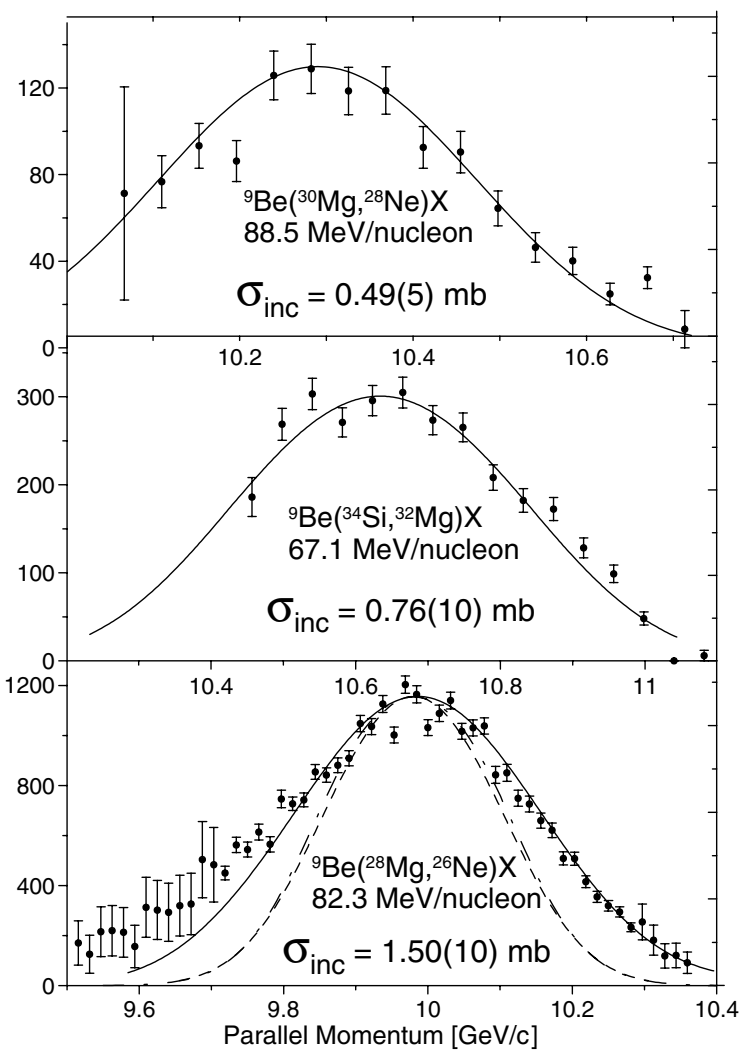

FIG. 1. Parallel-momentum distributions for two-proton knockout reactions. The theoretical curves include the Lorentz correction and the broadening arising mainly from the target thickness. The full drawn curves are estimates for knockout of two protons in $0 d$ states. For ${ }^{28} \mathrm{Mg}$ the width (FWHM) without the stopping power correction would be $380 \mathrm{MeV} / c$; the comparison value for two protons in $1 s$ states (dashed) is $282 \mathrm{MeV} / c$ and for a single $0 d$ knockout (dashdotted) $298 \mathrm{MeV} / c$.

system. The full-energy peak efficiencies obtained this way ranged from $3.2 \%$ at $0.885 \mathrm{MeV}$ to $1.8 \%$ at $2.02 \mathrm{MeV}$. The resolution of approximately $2.5 \%$ was limited by the close distance of the detectors, by the differential energy loss between projectile and fragment, which differ in $Z$ by two units, and by the size of the target. The main states identified in Fig. 2 are all known from previous experiments, the $2^{+}$states from Coulomb excitation (see Ref. [11] and references therein) and the $4^{+}$states from in-beam gamma spectroscopy $[18,19]$. For the detailed analysis of the ${ }^{28} \mathrm{Mg}$ results it is important that a $10 \%$ excess of intensity near $1.7 \mathrm{MeV}$ must be due to the second $2^{+}$and $0^{+}$states, decaying by gamma rays of 1.66 and $1.80 \mathrm{MeV}$ [18] and seen also in beta decay [20]. The reaction of ${ }^{34} \mathrm{Si}$ shows an apparent excess of gamma intensity above the adjusted continuum curve for energies above $1.4 \mathrm{MeV}$. This suggests that the cross section is fragmented to unobserved higher states and that it is not safe to deduce cross sections from an intensity balance. For ${ }^{32} \mathrm{Mg}$ (not shown in the figure) the statistics was low;

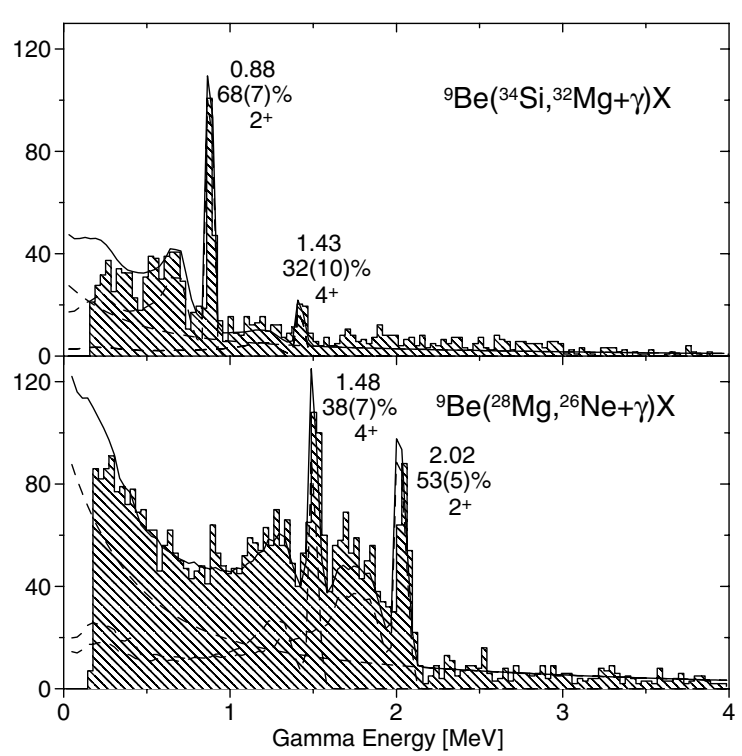

FIG. 2. Gamma-ray spectra in counts per $32 \mathrm{keV}$ bins. The main peaks, all representing $2^{+} \rightarrow 0^{+}$and $4^{+} \rightarrow 2^{+}$transitions, are labeled by the energy in $\mathrm{MeV}$ (uncertainty $0.02 \mathrm{MeV}$ ) and by the absolute intensity relative to the number of observed fragments. The dashed peak shapes are simulated response curves normalized to match the number of counts in the fullenergy peaks. The continuous distribution (dashed line) is attributed to radiations from the target and is adjusted to the ${ }^{28} \mathrm{Mg}$ data as a sum of a straight line and an exponential. The same continuum, scaled with the number of residues, is shown in the ${ }^{34} \mathrm{Si}$ spectrum. The full-drawn lines represent the sum of all three components.

the two main peaks were $1.32 \mathrm{MeV}\left(2^{+}\right), 53(13) \%$ and $1.73 \mathrm{MeV}\left(4^{+}\right), 39(13) \%$. In the following we restrict the analysis of the partial cross sections to the case of ${ }^{28} \mathrm{Mg}$, for which the gamma information appears close to complete. This is also the case that is simplest from a theoretical viewpoint since both the initial and final states have a spherical configuration stabilized by the pronounced subshell closure at $N=16$ [12]. The energies agree well with a calculation using the $s d$ model space with the USD effective interaction [21,22]; see Table I.

A comprehensive theory for direct two-proton knockout will necessarily be more complex than that for knockout of a single nucleon. In the latter case the partial cross

TABLE I. Theoretical spectroscopic factors $S_{p}\left(I^{\pi}\right)$ in the $d_{5 / 2}^{p}$ single subshell model (left) and comparison (right) of the experimental values with an $s d$ shell-model calculation for the case of ${ }^{28} \mathrm{Mg}$. $S_{\mathrm{ex}}$ is in units of $\sigma_{22}=0.29 \mathrm{mb}$.

\begin{tabular}{cccccccc}
\hline \hline$I^{\pi}$ & $p=2$ & $p=4$ & $p=6$ & $E_{\text {th }}$ & $E_{\text {ex }}$ & $S_{\text {rel }}$ & $S_{\text {ex }}$ \\
\hline $0^{+}$ & 1 & 1.33 & 1 & 0.00 & 0.00 & 1.6 & $2.4(5)$ \\
$2^{+}$ & 0 & 1.67 & 5 & 2.01 & 2.02 & 0.14 & $0.3(5)$ \\
$4^{+}$ & 0 & 3 & 9 & 3.66 & 3.50 & $(2.0)$ & $2.0(3)$ \\
$2^{+}$ & $\cdots$ & $\cdots$ & $\cdots$ & 3.45 & 3.7 & 0.46 & $0.5(3)$ \\
\hline \hline
\end{tabular}


section for a given $j$ channel factorizes into a part describing the contribution from many-body nuclear structure ("the spectroscopic factor") and a part describing the reaction dynamics ("the single-particle cross section") [4]. This no longer holds for the two-nucleon process. For this, many two-particle components may, within each total-angular-momentum channel, contribute coherently. The transition amplitude can then have significant interference effects reflecting combined contributions from structure and reaction geometry. One may think of the ideal theory as one uniting many-body shellmodel wave functions and the eikonal approximation with the geometrical explicitness of few-body reaction models. Such a theory does not exist. In what follows, we successively introduce four simple approximations in order to circumscribe the problem and arrive at a first quantitative understanding.

(i) Calculation of the absolute cross section in eikonal reaction theory assuming two uncorrelated protons. For a process engaging any two particles in the valence shell it is convenient to assume that the two particles are uncorrelated. Eikonal reaction theory [4] then gives the basic unit of cross section as (neglecting spin-orbit splitting)

$\sigma_{\ell_{1} \ell_{2}}=\int d \vec{b}\left|S_{c}\right|^{2} \prod_{i=1,2} \frac{1}{2 \ell_{i}+1} \sum_{m_{i}}\left\langle\ell_{i} m_{i}\left|\left(1-\left|S_{p i}\right|^{2}\right)\right| \ell_{i} m_{i}\right\rangle$.

Here $S_{\mathrm{c}}$ and $S_{p}$ are the elastic $S$ matrices (profile functions) for the core- and proton-target systems, functions of their individual impact parameters, and are calculated in the optical limit of Glauber theory. The individual proton-core relative motion wave functions $|\ell m\rangle$ are calculated in Woods-Saxon potentials with depths adjusted to reproduce the proton separation energies. We neglect recoil of the heavy core. Equation (1) allows a simple interpretation as the integral over the two-dimensional impact parameter and average over $m$ substates of the joint probability of the core being elastically scattered and of the two protons being absorbed. Diffractive breakup processes are assumed to be negligible for these very deeply bound protons. (For the simpler case of diffractive breakup of a single proton, a sum-rule estimate gives an upper limit of 20\%; the contribution is most likely smaller in the two-proton case.) For ${ }^{28} \mathrm{Mg}$ the cross section $\sigma_{22}$ is $0.29 \mathrm{mb}, \sigma_{00}$ is $0.35 \mathrm{mb}$, and $\sigma_{20}$ is $0.32 \mathrm{mb}$. The corresponding $\ell=2$ (0) single-particle (unit) cross sections for knockout of one proton are 11.6 (14.8) mb. For ${ }^{30} \mathrm{Mg} \sigma_{22}$ is $0.22 \mathrm{mb}$, and for ${ }^{34} \mathrm{Si} \sigma_{22}$ is $0.144 \mathrm{mb}$.

In order to obtain reaction cross sections from the unit values, we need the equivalent of a spectroscopic factor. From the approximation of no correlations it follows from simple combinatorics that for $p$ particles in the valence shell this factor is $S_{p}=p(p-1) / 2$. Thus for ${ }^{28} \mathrm{Mg}$ with $p=4$ we obtain $6 \sigma_{22}=1.8 \mathrm{mb}$, measured value $1.50(10) \mathrm{mb}$, for ${ }^{34} \mathrm{Si}$ with $p=6$ we obtain
$2.2 \mathrm{mb}$, measured value $0.76(10) \mathrm{mb}$, and for ${ }^{30} \mathrm{Mg}$ with $p=4$ we obtain $1.3 \mathrm{mb}$, measured value $0.49(5) \mathrm{mb}$. In view of the approximations made, all three cases must be considered to show good agreement between experiment and the direct-reaction model, demonstrating that we have, at least, a semiquantitative understanding of the absolute cross sections. It is tempting to speculate that the lower values measured for two of the cases could reflect the coexistence in these nuclei of deformed and spherical configurations with an appreciable fraction of the strength spread to states above the neutron threshold. More realistically, however, it should be kept in mind that short-range correlations, not included in the shell-model description, recently have been shown [23] to reduce the cross sections by a factor $R_{s}=0.5-0.6$ for a single deeply bound proton or neutron. For the case of two protons a reduction by the square of this might then be a reasonable expectation.

The same calculation has been applied to the reaction ${ }^{9} \mathrm{Be}\left({ }^{11} \mathrm{Be},{ }^{8} \mathrm{He}+n\right) X$ [10] proceeding between two $s$ states for the odd neutron. The calculated unit cross section from Eq. (1) is $1.05 \mathrm{mb}$. With two protons in the $p$ shell for ${ }^{11} \mathrm{Be}$ one has $S_{2}=1$ (Table I). However, this experiment detected only the $s$ state, which has an occupancy of $74 \%$ in the initial state, and there is further a $10 \%$ reduction arising from recoil effects [10]. The calculation thus gives a partial cross section of $0.7 \mathrm{mb}$ in reasonable agreement with the measured approximate value of $0.35 \mathrm{mb}$ for this broad continuum state.

(ii) Same approximation for the momentum distribution. The observation of parallel-momentum distributions centered close to beam velocity in Fig. 1 is, qualitatively, the equivalent of the strongly forward-peaked angular distributions that identify low-energy transfer reactions as direct. To turn the observed momentum shapes and widths into a quantitative test, we need the differential equivalent of Eq. (1). The calculation, however, can be simplified by noting that the momentum distributions probe the momentum content in the surface of the nucleus and that this quantity varies slowly with distance from the core. Therefore, in the uncorrelated approximation, the distribution for two independent particles is simply given by the convolution of the separate distributions for the two nucleons as shown in Fig. 1. The curves confirm that we are dealing with the direct knockout of two $0 d$ protons as assumed in (i). Other interpretations, shown in the figure, agree less well. The momentum distributions are not very distinct. Furthermore, in a more realistic theory several different $j$ pairs will contribute to each angular-momentum channel, so the momentum distributions are not expected to have the same diagnostic value as they have for the one-nucleon process [3,5-7].

(iii) Uncorrelated approximation assuming a single active $j$ shell. With this simplification we can calculate spectroscopic factors to individual excited levels. For the even- $Z$ elements neon to silicon it is a good first 
approximation to assume that all valence protons numbering $p=2,4,6$ are in the $0 d_{5 / 2}$ subshell. The only possible final states now have $I=0,2,4^{+}$. The corresponding spectroscopic factors $S_{p}\left(I^{\pi}\right)$ are given in Table I. Note that they sum to the inclusive $S_{p}$ given by the formula in (i) and that the highest possible spin is favored. The values for $p=4$ are in qualitative agreement with results for ${ }^{28} \mathrm{Mg}$, obtained by dividing the partial cross sections obtained from an input-output intensity balance by the unit cross section of Eq. (1). However, this model fails to explain the low cross section to the $2^{+}$state. This suggests a fourth approximation.

(iv) Full diagonalization in the $s d$ shell, simplified reaction model. Assume now that the two protons which participate in the reaction are those with $0 s$ internal (relative) motion. This model has been applied to diproton decay [24], and it is an appropriate assumption for two-nucleon transfer reactions such as $(p, t)$ [25]. It cannot be expected to give quantitative results in the present case, and we tentatively interpret the outcome as a relative spectroscopic factor. This is obtained by projecting the shell-model two-particle overlap obtained with a complete $s d$-shell wave function onto a twoparticle wave function which represents the two protons correlated with $\ell=0$ for the relative motion with total spin $S=0$ and total isospin $T=1$. Using the formalism developed in [26] the relative spectroscopic factor is $S_{\text {rel }}=\left|\left\langle\Psi(A-2, Z-2) \times \psi_{c} \mid \Psi(A, Z)\right\rangle\right|^{2}$, where $\Psi$ are the $s d$-shell wave functions and $\psi_{c}$ is the diproton cluster wave function. The di-proton spectroscopic factors normalized to the experimental value for the $4^{+}$state are given in Table I. They are in qualitative agreement with experiment; in particular, we note that in this truncated limit the reduced cross section to the first $2^{+}$state can be understood.

In conclusion, we have shown that when a neutron-rich projectile reacts with a light nuclear target, the knockout of two protons occurs as a direct reaction. Consequently, the observed partial cross sections to individual final levels convey selective information about nuclear structure. We expect the same to be the case for two-neutron knockout from a proton-rich nucleus. More experiments and also the development of a more complete reaction theory are interesting challenges. The experimental results suggest that the pattern and absolute magnitudes of the partial cross sections will provide specific information on the detailed nature of the states involved. This calls to mind the "fingerprints" characteristic of one- and two-nucleon transfer reactions on complex nuclei; see Ref. [27]. It is interesting to note that, measured from beta stability $\left({ }^{31} \mathrm{P}\right),{ }^{28} \mathrm{Mg}$ is less than half way to the drip line $\left({ }^{22} \mathrm{C}\right)$. This implies that this new direct reaction will be a viable method in a wide region extending to the drip line.

This work was supported by the U.S. National Science Foundation under Grants No. PHY-01 10253 and No. PHY0070911 and by the United Kingdom Engineering and Physical Sciences Research Council (EPSRC) Grant No. GR/M82141.

*Present address: Institut für Kernphysik, Technische Universität Darmstadt, Germany.

†Electronic address: hansen@nscl.msu.edu

[1] G. R. Satchler, Direct Nuclear Reactions (Oxford University Press, Oxford, 1983), pp. 1-833.

[2] H. Feshbach, Theoretical Nuclear Physics: Nuclear Reactions (Wiley, New York, 1992), p. 455.

[3] A. Navin et al., Phys. Rev. Lett. 81, 5089 (1998).

[4] J. A.Tostevin, J. Phys. G 25, 735 (1999).

[5] V. Maddalena et al., Phys. Rev. C 63, 024613 (2001).

[6] J. Enders et al., Phys. Rev. C 65, 034318 (2002).

[7] P. G. Hansen and B. M. Sherrill, Nucl. Phys. A693, 133 (2001).

[8] K. Yoneda et al., Phys. Lett. B 499, 233 (2001).

[9] G. Astner and G. K. Wolf, Nucl. Phys. A147, 481 (1970).

[10] L. Chen et al., Phys. Lett. B 505, 21 (2001); L. Chen, Ph.D. thesis, Michigan State University, 2000.

[11] B.V. Pritychenko et al., Phys. Lett. B 461, 322 (1999); 467, 309(E) (1999).

[12] T. Otsuka et al., Phys. Rev. Lett. 87, 082502 (2001).

[13] B. A. Brown, Prog. Part. Nucl. Phys. 47, 517 (2001).

[14] D. J. Morrissey et al., Nucl. Instrum. Methods Phys. Res., Sect. B 204, 90 (2003).

[15] D. Bazin et al., Nucl. Instrum. Methods Phys. Res., Sect. B 204, 629 (2003).

[16] W. F. Mueller et al., Nucl. Instrum. Methods Phys. Res., Sect. A 466, 492 (2001).

[17] GEANT-detector description and simulation tool, version 3.21, CERN Program Library Long Writeup W5013, 1994.

[18] O. Sorlin et al., Nucl. Phys. A685, 186 (2001).

[19] F. Azaiez et al., Eur. Phys. J. A 15, 93 (2002).

[20] A. T. Reed et al., Phys. Rev. C 60, 024311 (1999).

[21] B. A. Brown and B. H. Wildenthal, Annu. Rev. Nucl. Part. Sci. 38, 29 (1988); B. H. Wildenthal, Prog. Part. Nucl. Phys. 11, 5 (1984).

[22] B. A. Brown, http://www.nscl.msu.edu/ brown/sde.htm

[23] B. A. Brown, P. G. Hansen, B. M. Sherrill, and J. A. Tostevin, Phys. Rev. C 65, 061601(R) (2002).

[24] B. A. Brown, Phys. Rev. C 43, R1513 (1991); 44, 924 (1991).

[25] N. K. Glendenning, At. Data Nucl. Data Tables 16, 1 (1975).

[26] N. Anyas-Weiss et al., Phys. Rep. 12, 201 (1974).

[27] Aa. Bohr and B. R. Mottelson, Nuclear Structure Vol. 2 (Benjamin, New York, 1975), pp. 258-261, 646. 